\title{
Beyond Electrochemical Analysis: 2D to 4D Correlation of Structure and Chemistry in Li-ion Batteries
}

\author{
Stefanie Freitag ${ }^{1}$ \\ ${ }^{1}$ Carl Zeiss Microscopy GmbH, Market Segment Material Sciences, Munich Site, Germany
}

The breakthrough of the li-ion technology was initiated when Sony started the production of the first commercial available li-ion batteries (LIB) with graphite as anode material and $\mathrm{LiCoO}_{2}$ as cathode material [1]. Current battery research activities are often focused on NMC batteries $\mathrm{Li}\left(\mathrm{Ni}_{\mathrm{x}}, \mathrm{Mn}_{\mathrm{y}}, \mathrm{Co}_{\mathrm{z}}\right) \mathrm{O}_{2}$. During charging lithium ions move from the cathode through the separator to the anode, where they are intercalated into the carbon layers of the graphite. Intercalation can lead to volume expansions of the actual material and reaction processes between for example lithium and electrolyte cause layer formations in the active material. Life time, capacity and especially aging effects in the LIB are mainly determined by the constituent active materials and the microstructure of the active mass coating. Important features to be analyzed and controlled are particle size, shape and distribution, the presence of voids and defects within the particles, porosity, pore tortuosity, and coating thicknesses. [2].

Pure electrochemical analysis methods like generating an IU characteristic and impedance or gravimetric measurements can lead to wrong battery failure interpretations. Recent reports have shown that the correlation of chemical and microstructure analysis allows the interpretation of for example impedance changes and conductivity losses. With this information researcher are enabled to understand the root cause of defective batteries. As a result targeted optimization of their lithium ion battery production and preparation process is possible.

This study explains in a first step the benefits of correlating light microscopy data with integrated Raman spectroscopy in an scanning electron microscope (SEM) for LIB research. In many cases, two-dimensional microscopy studies are not sufficient for the full characterization of the LIB electrodes. This calls for a second step, a three-dimensional microscopy approach [2]. The experiments were done with a cycled and uncycled mobile phone battery as well as a 18650 battery.

It was shown that light microscopy offers information about grain sizes in the electrodes, degrading of several components through ablation and phase orientations in the anode, thus allows the selection of a region of interest. A correlative software enables the exact relocation of this area in the scanning electron microscope which delivers high resolution images of small cracks in particles as well as the non-conductive separator and binder. The binder material itself has a low conductivity, thus oversupply needs to be avoided [3]. Electron microscope images revealed an inhomogeneous binder distribution, that isn't evenly surrounding all particles. In separators, the alteration of polymer molecule orientations e.g. in polypropylene can cause property changes in various ways, described in many papers $[4,5,6]$. In-situ Raman spectroscopy adds information about organic and inorganic material compositions. With the SEM and in-situ Raman microscopy it was shown that all li-ion battery components can be analyzed including oxides, polymers, binder material and electrode material. It was found that separators can have different molecule orientations after cycling. Raman spectroscopy proved that several charge and discharge cycles influence the orientation from uniaxial to biaxial. The 3D X-ray microscope measurements added insights into pore distributions and tortuosity thus explaining parameter like conductivity loss and worse charge carrier transport.

Batteries can be imaged with various microscopic methods, each revealing a different information. All methods have benefits and disadvantages, therefore using these methods in a combination often 
leads to deeper understanding of the li-ion battery properties. Obvious reasons to use microstructure analysis methods on top of electrochemical metrology are provided. Suitable parameter for battery imaging are discussed. This forward-looking solution visualizes new insights into the battery characteristics. It allows the more reliable interpretation of performance parameter through correlating different analysis methods.

\section{References:}

[1] B. Ketterer et al, Forschungszentrum Karlsruhe GmbH, FZKA 7503, (2009) p.69.

[2] C. Weisenberger et al, Microscopy\&Analysis, (2015) p.17-19.

[3] P. Voelker, Thermo Fisher Scientific, R\&D Magazine, (2014) p.1-5.

[4] S. Rettenberger et al, Rheol Acta, (2002) p.332-336.

[5] C.P. Lafrance et al , Polymer, 35(18), (1994) p.3927-3935.

[6] J.H. Magill, et al, Int. Polym. Process., (1987) p.66-76.

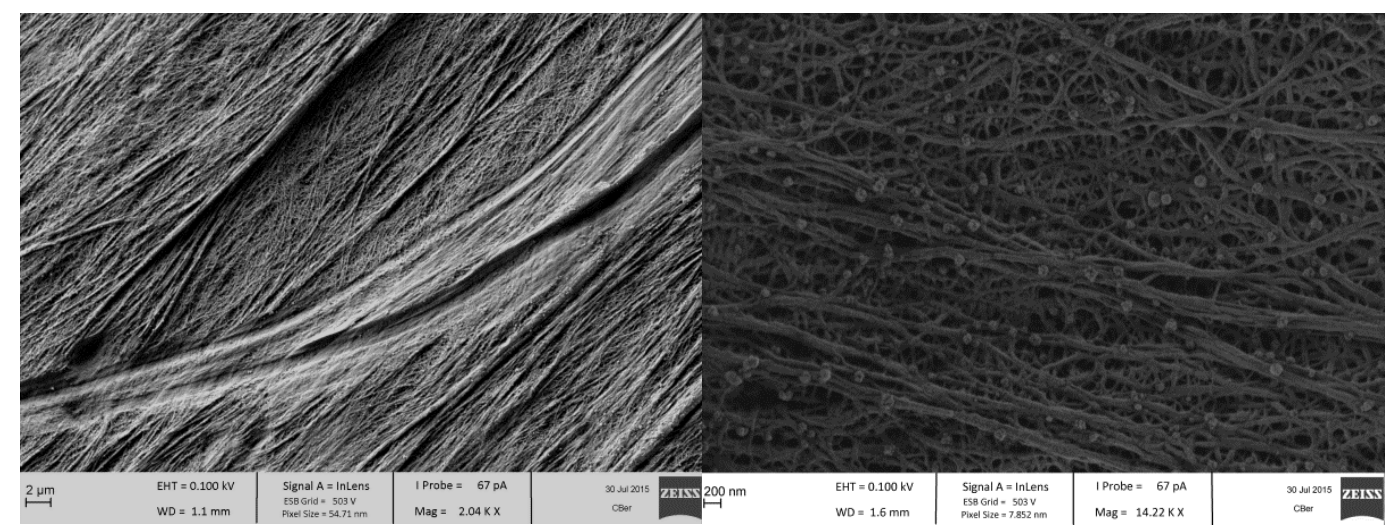

Figure 1. SEM images of a li-ion battery separator without observable charging effects although no conducting coating was used. The separator is a polymer and as shown here consists of molecules aligned in fibers.

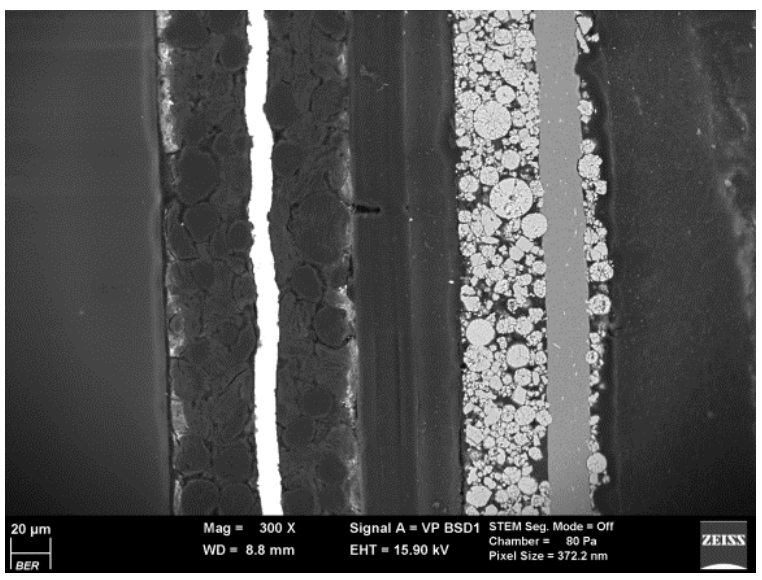

Figure 2. SEM image revealing cracks in particles and degradation effects in the separator Sample courtesy of Christina Berger, Carl Zeiss Microcopy GmbH

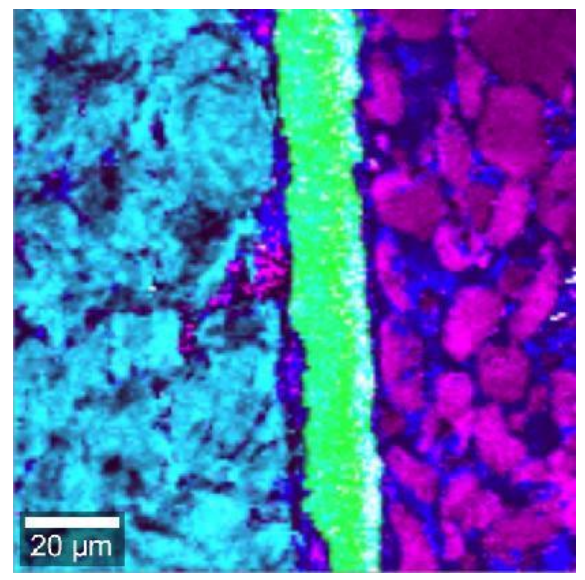

Figure 3. Raman microscopy indicating polyethylene as material of the separator. Sample courtesy from Witec GmbH. 